\title{
High frequency of hybrid Escherichia coli strains with combined Intestinal Pathogenic Escherichia coli (IPEC) and Extraintestinal Pathogenic Escherichia coli (ExPEC) virulence factors isolated from human faecal samples
}

\author{
Bjørn-Arne Lindstedt ${ }^{*}$ (D), Misti D. Finton ${ }^{1}$, Davide Porcellato ${ }^{1}$ and Lin T. Brandal ${ }^{2}$
}

\begin{abstract}
Background: Classification of pathogenic Escherichia coli (E. coli) has traditionally relied on detecting specific virulence associated genes (VAGs) or combinations thereof. For E. coli isolated from faecal samples, the presence of specific genes associated with different intestinal pathogenic pathovars will determine their classification and further course of action. However, the E. coli genome is not a static entity, and hybrid strains are emerging that cross the pathovar definitions. Hybrid strains may show gene contents previously associated with several distinct pathovars making the correct diagnostic classification difficult. We extended the analysis of routinely submitted faecal isolates to include known virulence associated genes that are usually not examined in faecal isolates to detect the frequency of possible hybrid strains.
\end{abstract}

Methods: From September 2012 to February 2013, 168 faecal isolates of E. coli routinely submitted to the Norwegian Institute of Public Health (NIPH) from clinical microbiological laboratories throughout Norway were analysed for 33 VAGs using multiplex-PCR, including factors associated with extraintestinal pathogenic $E$. coli (ExPEC) strains. The strains were further typed by Multiple Locus Variable-Number Tandem-Repeat Analysis (MLVA), and the phylogenetic grouping was determined. One isolate from the study was selected for whole genome sequencing (WGS) with a combination of Oxford Nanopore's MinION and Illumina's MiSeq.

Results: The analysis showed a surprisingly high number of strains carrying ExPEC associated VAGs and strains carrying a combination of both intestinal pathogenic E. coli (IPEC) and ExPEC VAGs. In particular, 93.5\% (101/ 108) of isolates classified as belonging to an IPEC pathovar additionally carried EXPEC VAGs. WGS analysis of a selected hybrid strain revealed that it could, with present classification criteria, be classified as belonging to all of the Enteropathogenic Escherichia coli (EPEC), Uropathogenic Escherichia coli (UPEC), Neonatal meningitis Escherichia coli (NMEC) and Avian pathogenic Escherichia coli (APEC) pathovars.

Conclusion: Hybrid EXPEC/IPEC E. coli strains were found at a very high frequency in faecal samples and were in fact the predominant species present. A sequenced hybrid isolate was confirmed to be a cross-pathovar strain possessing recognised hallmarks of several pathovars, and a genome heavily influenced by horizontal gene transfer.

Keywords: Escherichia coli, Pathogenic, ExPEC, IPEC, Hybrid strains, MinION

\footnotetext{
* Correspondence: bjorn-arne.lindstedt@nmbu.no

${ }^{1}$ Faculty of Chemistry, Biotechnology and Food Science, Norwegian

University of Life Sciences, P.O. Box 5003, N-1432 Ås, Norway

Full list of author information is available at the end of the article
}

(c) The Author(s). 2018 Open Access This article is distributed under the terms of the Creative Commons Attribution 4.0 International License (http://creativecommons.org/licenses/by/4.0/), which permits unrestricted use, distribution, and reproduction in any medium, provided you give appropriate credit to the original author(s) and the source, provide a link to the Creative Commons license, and indicate if changes were made. The Creative Commons Public Domain Dedication waiver (http://creativecommons.org/publicdomain/zero/1.0/) applies to the data made available in this article, unless otherwise stated. 


\section{Background}

Escherichia coli (E. coli) is a highly diverse and predominant species among facultative anaerobic bacteria of the human gastrointestinal tract [1]. E. coli comprises non-pathogenic commensals as well as strains causing a range of diseases. E. coli strains capable of causing extraintestinal infections are designated as extraintestinal pathogenic E. coli (ExPEC) to distinguish them from strains causing intestinal disease, commonly designated as intestinal pathogenic E. coli (IPEC).

ExPEC can cause a wide variety of extraintestinal infections at multiple anatomical sites. ExPEC frequently cause urinary tract infection (UTI), septicaemia, meningitis, as well as causing soft tissue damage $[2,3]$. ExPEC includes, among others, the pathovars uropathogenic $E$. coli (UPEC) associated with urinary tract infection in human and animals, neonatal meningitis-associated $E$. coli (NMEC), septicaemic E. coli (SePEC) causing systemic infection in human and animals, avian pathogenic E. coli (APEC) that cause avian colibacillosis, and a potentially emerging ExPEC lineage named endometrial pathogenic E. coli (EnPEC) $[4,5]$.

A wide range of VAGs have been associated with ExPEC and common virulence attributes among ExPEC strains are those enabling their extraintestinal lifestyle e.g. genes coding for the production of adhesins, toxins, protectins, siderophores, iron transport systems, and invasins $[2,6-9]$. It is believed that ExPEC are facultative pathogens, which reside in the normal gut flora as commensals in some groups of the healthy population [8]. However, there are no universal accepted concrete genetic criteria for defining an E. coli strain as ExPEC nor for definite pathovar classification within the ExPEC group. Thus, the true pathovar classification can only be done on the basis of the isolation source for the majority of ExPECs.

There is limited information regarding the frequency of ExPEC strains in the human intestine, however a recent meta study of more than 500 published papers assessed a prevalence of ExPEC strains among faecal isolates of about $10 \%$ in healthy individuals [10]. Reference laboratories or diagnostic microbiological laboratories routinely search for only the established IPEC virulence factors in faecal samples from symptomatic patients. There exist little data on the frequency of ExPEC related virulence factors among these strains.

The aim of this study was to investigate the frequency and combination of virulence markers including VAGs used for IPEC pathovar classification and a selection of VAGs related to ExPEC pathovars among $E$. coli strains submitted from individuals showing signs of gastrointestinal infections. We assessed the frequency of ExPEC and IPEC strains, phylogenetic grouping and the MLVA-genotype.
In light of the large German O104:H4 outbreak in 2011 [11], which was caused by a hybrid Enteroaggregative E. coli (EAEC)/Shiga toxin producing E. coli (STEC) strain [12], the monitoring of isolates to detect new or altered combinations of VAGs is important as it may give a pre-warning of emerging strains harbouring novel VAG combinations, which should be studied in closer detail to assess whether they also have altered virulence capabilities.

\section{Methods}

\section{Bacterial isolates}

All 168 E.coli strains were obtained from the culture collection at the National Reference Laboratory for Enteropathogenic Bacteria at the Norwegian Institute of Public Health (NIPH).

\section{VAG PCR \\ ExPEC VAGs, cnf1, cnf2, cnf3, ehaA and ehaG PCR}

PCR-primers for amplication of the following VAGs were constructed using primer3 software (http://www. ncbi.nlm.nih.gov/tools/primer-blast/) and DNASTAR's Lasergene software module "Primer Select" (DNAstar, Inc., Madison, WI): cytotoxic necrotising factors 1-3 cnf1, cnf2, cnf3; autotransporters (ATs) sat, tsh, vat, ehaA, and ehaG; iron acquisition iutA, sitA, iucD, iroC, $f b p B$, and $f y u A$; adhesins $s f a S$, papC, and tosA; protectins kpsS, traT and iss; the invasin gene $i b e A$, and primers directed at orf5 in the gimB genetic island (sequence acc. no. AY170898). Primers directed at the etsA gene encoding the macrolide-specific efflux protein EtsA were also designed (see Additional file 1). PCR primers aimed at ExPEC VAGs and ehaA + ehaG were combined in four multiplex reaction mixes as follows: Multiplex 1 (cnf1, cnf2, cnf3, iutA, ibeA and sitA), Multiplex 2 (iucD, iss, traT, iroC, sat, papC and ehaA), Multiplex 3 (tsh, gimB-genetic island, etsA, kpsS and sfaS), and Multiplex 4 (tos $A, v a t, f b p B$, fyuA and ehaG).

All primers had a final concentration of $5 \mu \mathrm{M}$. The PCR was run on a GeneAmp 9700 thermocycler (Applied-Biosystems, Foster City, CA, USA) with the following conditions: multiplexes 1,2 and $4 ; 95{ }^{\circ} \mathrm{C}$ for $15 \mathrm{~min}$, then 25 cycles of $94{ }^{\circ} \mathrm{C}$ for $30 \mathrm{~s}, 58{ }^{\circ} \mathrm{C}$ for $90 \mathrm{~s}$ and $72{ }^{\circ} \mathrm{C}$ for $90 \mathrm{~s}$, followed by a hold on $72{ }^{\circ} \mathrm{C}$ for $10 \mathrm{~min}$ after temperature cycling has ended. Multiplex 3; $95{ }^{\circ} \mathrm{C}$ for $15 \mathrm{~min}$, then 25 cycles of $94{ }^{\circ} \mathrm{C}$ for $30 \mathrm{~s}, 60{ }^{\circ} \mathrm{C}$ for $90 \mathrm{~s}$ and $72{ }^{\circ} \mathrm{C}$ for $90 \mathrm{~s}$, followed by a hold on $72{ }^{\circ} \mathrm{C}$ for $10 \mathrm{~min}$ after temperature cycling has ended. The multiplexes were diluted 1:25 and run in separate capillaries on an ABI 3130 Genetic Analyzer (Applied-Biosystems, Foster City, CA, USA) with GS 600LIZ as internal size standard. 


\section{IPEC VAGs}

PCR for detecting common IPEC VAGs was performed as previously published [13-15]. In all, primers for the following IPEC VAGs were included: stx1, stx2, eaeA, ipaH, LTI, STIa, STIb, aggR, ehxA, bfp with $16 \mathrm{~S}$ control rrs (see Additional file 1).

\section{Phylogenetic group PCR}

The improved phylogenetic PCR-assay [16] of the original assay described by Clermont [17] was used to assign the $E$. coli isolates to major phylogenetic groups and subgroups.

\section{MLVA}

Multi-locus variable-number tandem repeats analysis was performed using a modified version of the 10-loci generic E. coli MLVA scheme previously published [18]. The PCR-amplicon of the published CCR001 locus contains two variable repeated elements, and the modified scheme allows typing of both these variable elements increasing the number of the generic E. coli MLVA to 11-loci. The modification consists of a change of dyes and an additional new reverse-primer at the CCR001 locus as follows: the 6FAM dye was removed from the published CCR001 forward primer [18] and the published unlabelled CCR001 reverse primer was labelled with 6FAM and renamed CCR001aR. A new second VIC-labelled reverse primer was added “CCR001bR: 5' VIC-CGCATTTTATCTGTCTGTACGGC - 3”'. The combination of both reverse primers made it possible to simultaneously separate both repeat containing regions at the CCR001 locus.

\section{Stx subtyping}

Subtyping of stx 1 and stx 2 was performed as described in Brandal et al. 2015 [15].

\section{Oxford Nanopore MinION sequencing}

The hybrid ExPEC/IPEC strain FHI_NMBU_03 identified by PCR, was chosen for sequencing by the MinION MK1 device. DNA was quantified using the Qubit fluorometer (Life Technologies, Paisley, UK) and $200 \mathrm{ng}$ of DNA was used for library preparation. The strain was sequenced using the R9.4 SpotON flow cell and the SQK-RAD002 rapid sequencing kit. All runs were prepared according to the standard protocol of Oxford Nanopore Technologies (Oxford, UK). The flow cells were primed with a priming solution that consisted of a mixture of nuclease free water and Fuel Mix. The library was then loaded into the MinION SpotON port and the 48-h sequencing protocol was selected in the MinKNOW software. The basecalling was done through the Metrichor Desktop Agent using 1D Basecalling for the SQK-RAD002 protocol.

\section{Illumina MiSeq sequencing}

Illumina sequencing was performed on an Illumina MiSeq platform (Illumina Inc., San Diego, CA, USA). Library was prepared using the Nextera XT kit (Illumina Inc) according to manufacturer's instructions and was sequenced using a 300 bp paired-end sequencing kit (Illumina Inc).

\section{Sequence analysis}

Raw Illumina reads were paired and quality filtered using Trimmomatic [19] and bases with low quality $(<\mathrm{q} 20)$ were discarded. MinION reads were extracted using poRe [20] and both read types were assembled using SPAdes [21] version 3.5.0 using the option "--nanopore".

Using combined MiSeq and MinION data, the sequences were assembled into a large contig constituting the genome and a contig containing a large virulence plasmid.

The sequence data was annotated using four different services, the NCBI Prokaryotic Genome Annotation Pipeline [22], the BASys Bacterial Annotation System [23], The RAST Annotation Server [24] and Prokka [25]. The sequences were further analysed using a variety of free and publicly available software. Integrated prophages and genomic islands (GIs) were searched using PHASTER [26] and Island Viewer 4 [27] respectively, and the final location of prophages and GIs was determined using a combination of the resulting data. Multilocus sequence typing (MLST)-type, Fim-type, antibiotic resistance genes, and virulence genes were searched using online services from the Center for Genomic Epidemiology (CGE) at the Danish Technical University (DTU), Lyngby, Denmark (http://www.genomicepide miology.org/). Assembly and annotation of the isolate FHI_NMBU_03 and its plasmid are publicly available at NCBI (accession number CP019455 and CP019456, respectively).

\section{Results \\ PCR}

The pathovar distribution among the $168 \mathrm{E}$. coli faecal isolates were as follows: 53 non-IPEC (31.5\%), (including 2 strains harbouring ehaG only and 1 strain negative for all tested VAGs). One hundred eight IPEC (64.3\%), (including 49 atypical-EPEC (aEPEC) (29.2\%), 31 STEC (18.5\%), 21 enterotoxigenic Escherichia coli (ETEC) (12.5\%), 7 necrotoxin producing E. coli (NTEC) (4.2\%), 3 enteroinvasive Escherichia coli (EIEC) (1.8\%), 2 EAEC (1.2\%), 1 typical-EPEC (tEPEC) (0.6\%), and 1 STEC/ ETEC (stx2d, LTI, iss, traT and ehaG) hybrid strain $(0.6 \%))$. A total of 108 isolates (64.3\%) contained both recognised IPEC and ExPEC VAGs, thus 93.9\% (108/ $115)$ of the IPEC isolates also carried ExPEC VAGs. Fifty isolates (29.7\%) carried only recognised ExPEC VAGs without any accompanying IPEC associated genes (Table 1). The frequency of the phylogenetic subgroups 
Table 1 Distribution of pathotypes in E. coli faecal isolates

\begin{tabular}{llllll}
\hline Pathotype & EXPEC $^{\mathrm{a}}$ & All IPEC $^{\mathrm{b}}$ & Other $^{\mathrm{c}}$ & IPEC VF only $^{\mathrm{d}}$ & IPEC/EXPEC $^{\mathrm{e}}$ \\
\hline Number & 50 & 115 & 3 & 7 & 108 \\
Percent & $29.7 \%$ & $68.5 \%$ & $1.8 \%$ & $4.2 \%$ & $64.3 \%$
\end{tabular}

${ }^{a}$ Number of isolates with ExPEC VAGs only

${ }^{b}$ Number of isolates containing an IPEC VAG

'Two isolates positive for the ehaG gene only, and one isolate negative for al 33 markers

${ }^{\mathrm{d}}$ Number of isolates with IPEC VAGs exclusively

${ }^{\mathrm{e}}$ Number of isolates positive for combinations of both IPEC and ExPEC VAGs

were: 15 A0 (8.9\%), 35 A1 (20.8\%), 64 B1 (38.1\%), 7 B2_2 (4.2\%), 21 B2_3 (12.5\%), 20 D1 (11.9\%) and 6 D2 (3.6\%). The phylogenetic group distribution within each pathovar can be seen in Table 2. The highest frequency of combinatory IPEC/ExPEC strains was seen in phylogenetic subgroup B2_2 and group B1 (100 and 75\%, respectively). The frequency of the tested ExPEC related VAGs among all isolates can be seen in Table 3 . The ehaG gene was detected in $64.3 \%$ of the isolates and was the most common VAG in our collection. eae, ehaA, $e h x A$ and the gimB genetic island marker were present in $44.6,38.7,15.5$, and $1.2 \%$ of the isolates, respectively. When we looked at the average number of VAGs within all phylogenetic subgroups, we found that subgroup B2_2 carried most VAGs (7 VAGs) followed by B2_3 (6.9 VAGs), D2 (5.7 VAGs), D1 (5.4 VAGs), B1 (5.1 VAGs), A1 (4.1 VAGs), and A0 (3.3 VAGs).

The 168 isolates grouped into 131 different MLVAprofiles (1.23 isolates/MLVA-profile), where six clusters of identical MLVA-profiles containing three or more isolates were detected. Cluster 1 consisted of five ExPEC isolates of phylogenetic group A1, all from December 2012. Four of the isolates shared the same VAGs (sitA, iss, traT, kpsS and ehaG), while the fifth isolate had a deviating VAG composition (iutA, cnf2, iucD, iss, traT, $e h a A, f b p B$ and $e h a G$ ) and was designated NTEC due to the presence of the gene for cytotoxic necrotising factor

Table 2 Phylogenetic group distribution within each pathovar

\begin{tabular}{|c|c|c|c|c|c|c|c|}
\hline \multirow{2}{*}{$\begin{array}{l}\text { Phylogroup } \\
\text { Subgroup }\end{array}$} & \multicolumn{2}{|l|}{ A } & \multirow{2}{*}{$\begin{array}{l}\mathrm{B} 1 \\
\mathrm{~B} 1\end{array}$} & \multicolumn{2}{|l|}{ B2 } & \multicolumn{2}{|l|}{$\mathrm{D}$} \\
\hline & $\mathrm{AO}$ & $\mathrm{A} 1$ & & B2_2 & B2_3 & D1 & D2 \\
\hline aEPEC & 5 & 7 & 17 & 7 & 8 & 4 & 1 \\
\hline tEPEC & 0 & 0 & 0 & 0 & 1 & 0 & 0 \\
\hline STEC & 0 & 1 & 24 & 0 & 0 & 6 & 0 \\
\hline ETEC & 0 & 8 & 11 & 0 & 0 & 1 & 1 \\
\hline EAEC & 0 & 0 & 1 & 0 & 0 & 1 & 0 \\
\hline EIEC & 2 & 0 & 1 & 0 & 0 & 0 & 0 \\
\hline STEC/ETEC & 0 & 0 & 0 & 0 & 0 & 0 & 1 \\
\hline NTEC & 0 & 1 & 0 & 0 & 6 & 0 & 0 \\
\hline NON-IPEC & 8 & 18 & 10 & 0 & 6 & 8 & 3 \\
\hline$\Sigma$ Subgroups & 15 & 35 & 64 & 7 & 21 & 20 & 6 \\
\hline$\Sigma$ Phylogroups & \multicolumn{2}{|c|}{$50(29.8 \%)$} & 64 (30.1\%) & \multicolumn{2}{|c|}{$28(16.7 \%)$} & \multicolumn{2}{|c|}{26 (15.5\%) } \\
\hline
\end{tabular}

Table 3 Frequency of ExPEC associated virulence genes (PCR screening)

\begin{tabular}{|c|c|c|}
\hline $\begin{array}{l}\text { EXPEC associated } \\
\text { VAG }\end{array}$ & Comment & Frequency \\
\hline iss & Increased serum survival gene & $48.8 \%$ \\
\hline trat & $\begin{array}{l}\text { Gene encoding complement resistance } \\
\text { protein }\end{array}$ & $45.2 \%$ \\
\hline fyuA & $\begin{array}{l}\text { Ferric yersiniabactin uptake receptor } \\
\text { gene }\end{array}$ & $42.3 \%$ \\
\hline iucD & Aerobactin biosynthesis gene & $24.4 \%$ \\
\hline iutA & Ferric aerobactin receptor gene & $23.8 \%$ \\
\hline sitA & $\begin{array}{l}\text { Iron/manganese transport system } \\
\text { periplasmic binding protein gene }\end{array}$ & $23.2 \%$ \\
\hline kpsS & $\begin{array}{l}\text { Capsule polysaccharide export protein } \\
\text { gene }\end{array}$ & $18.5 \%$ \\
\hline tsh & $\begin{array}{l}\text { Temperature-sensitive hemagglutinin } \\
\text { autotransporter gene }\end{array}$ & $15.5 \%$ \\
\hline iroC & Salmochelin siderophore system gene & $12.5 \%$ \\
\hline vat & Vacuolating autotransporter toxin gene & $11.3 \%$ \\
\hline$f b p B$ & $\begin{array}{l}\text { Gene associated with urinary tract } \\
\text { infections }\end{array}$ & $10.1 \%$ \\
\hline sat & Secreted autotransporter toxin gene & $9.5 \%$ \\
\hline ibeA & Invasion protein gene & $8.3 \%$ \\
\hline etsA & Macrolide-specific efflux protein gene & $4.2 \%$ \\
\hline cnfl & $\begin{array}{l}\text { Gene encoding the cytotoxic necrotizing } \\
\text { factor } 1\end{array}$ & $3.6 \%$ \\
\hline sfas & S-fimbrial adhesin gene & $1.8 \%$ \\
\hline papC & $\begin{array}{l}\text { P-fimbriae outer membrane usher } \\
\text { protein gene }\end{array}$ & $1.8 \%$ \\
\hline $\operatorname{tos} A$ & Repeat-in-toxin gene & $1.8 \%$ \\
\hline cnf2 & $\begin{array}{l}\text { Gene encoding the cytotoxic necrotizing } \\
\text { factor } 2\end{array}$ & $0.6 \%$ \\
\hline cnf3 & $\begin{array}{l}\text { Gene encoding the cytotoxic necrotizing } \\
\text { factor } 3\end{array}$ & $N D^{a}$ \\
\hline
\end{tabular}

${ }^{\mathrm{a}}$ Not detected

2 (cnf2). Cluster 2 comprised of three aEPEC strains of phylogenetic group D1 isolated in October and November 2012, all of serogroup O55 with identical VAGs (eae, iss, ehaA, fbpB and ehaG). Cluster 3 contained six phylogenetic group B1 isolates from December 2012, where five isolates shared the same VAGs (LTI, iss, fyuA and ehaG) and was designated ETEC due to the presence of the LTI gene. Of these five isolates, four were serotyped into serogroup O78 while no serogroup could be assigned to the fifth isolate. The sixth isolate of MLVA-cluster 3 was also an O78 B1 isolate, but with different VAGs (sitA, iss, traT, kpsS, fyuA and ehaG). Cluster 4 consisted of four phylogenetic group B1 serogroup O103 STEC isolates from September to December 2012, all with identical VAGs (stx1a, eae, ehxA, traT, ehaA and $e h a G$ ). Cluster 5 consisted of six phylogenetic group B1 serotype O103:H2 STEC isolates from October and 
November 2012 submitted from the same Norwegian hospital with identical VAGs (stxla, eae eh $x A$, traT, ehaA and ehaG). Cluster 6 contained four phylogenetic group B2_2 aEPEC isolates from September and October 2012 where three of the isolates showed the same VAGs (eae, ibeA, iss, traT, iroC, tsh, vat and fyuA), while the fourth isolate had the following VAGs (eae, ibeA, tsh, vat and fyuA).

Among the 49 eae containing aEPEC isolates, the following VAGs were additionally detected: sitA, iss, ehaA, ehaG, papC, tsh, kpsS, vat, fyuA, iutA, iucD, fbpB, ehxA, sat, tsh, traT, ibeA, iroC, etsA, tosA, as well as a marker in the gimB genetic island. Only 4 of 49 aEPEC isolates (8.2\%) did not carry any VAGs previously associated with ExPEC strains. Thus, the majority (91.8\%) of our aEPEC faecal isolates contained VAGs related to ExPEC strains. The most common ExPEC related VAGs among the aEPEC isolates were: traT (49\%), iss (38.8\%), fyuA (32.7\%), tsh (26.5\%) and ibeA (26.5\%). When we divided the aEPEC isolates by phylogenetic group, we observed that the ibeA gene was present in $86.7 \%(13 / 15)$ of the aEPEC B2 strains, and the VAGs $e h a A$ and ehaG were also frequently present, 49 and $51 \%$ respectively.

The 31 STEC isolates contained 18 stx 1 only positive strains and 9 stx 2 only positive strains. The remaining four strains contained both $s t x 1$ and stx 2 . Among the STEC isolates, the following VAGs were additionally found: eae, iutA, iucD, iss, traT, iroC, ehaA, ehaG, etsA, fyuA, $k p s S$, ehxA and $f b p B$. The most common ExPEC related VAGs were: traT (58\%), iss (35.5\%), iucD (29\%) and iutA (25.8\%). Additional prevalent non-ExPEC factors present were: ehaA (96.8\%), ehaG (90.3\%), ehxA (74.2\%) and eae (71\%).

Among the 21 ETEC isolates, ehaG was detected in 12 strains $(57 \%)$, but ehaA was not detected in any of the ETEC isolates.

When we looked at pair-clustering of the VAGs we found that the most common pairs (in more than 20\% of isolates) of VAGs included: ehaA and ehaG in 60/168 (35.7\%) of the isolates, ehaG and traT or iss both combinations in 49/168 (29.2\%) of the isolates, eae and ehaA in $48 / 168(28.6 \%)$ of the isolates, eae and ehaG in $46 /$ $168(27.3 \%)$ of the isolates, iss and fyuA in $43 / 168$ (25.6\%) of the isolates, traT and eae or ehaA both combinations in $40 / 168(23.8 \%)$ of the isolates, iucD and iutA in 40/168 (23.8\%) of the isolates and traT and iss in $38 / 168(22.6 \%)$ of the isolates.

\section{Sequencing}

One strain from this study designated FHI_NMBU_03 from MLVA-cluster 6 was selected for whole genome sequencing using a combination of long- and short- read technologies, Oxford Nanopore MinION (91,865 reads) and Illumina MiSeq (361,031 reads), respectively. We were able to assemble a complete closed circular genome $(4,685,056$ bp acc. nr. CP019455) and a complete circular virulence plasmid (159,821 bp acc. nr. CP019456) pFHI_NMBU_03-1 from the combined runs. The genome sequence (coverage 21.6x) contained 4954 genes (gene density 1.057 genes/Kbp) and 200 pseudogenes, with a GC content of $51 \%$. The chromosome contains five integrated prophages according to PHASTER analysis [26], and 19 genomic islands (phages excluded) according to the Island Viewer 4 software [27]. FHI_NMBU_03 showed a surprising collection of both IPEC and ExPEC related VAGs as indicated by the PCR-analysis. It contained the locus of enterocyte effacement (LEE)-region of EPEC/EHEC as well as recognized markers for ExPEC subtypes of UPEC/APEC and NMEC. The LEE region of FHI_NMBU_03 contains 36 recognized genes, four open reading frames (ORFs) of unknown function as well as two pseudogenes, and is inserted in the selC tRNA gene. The eae-intimin subtype of FHI_NMBU_03 is $\beta 2$. The LEE-encoded Tir protein of FHI_NMBU_03 is, by BLAST search, identical to three Tir proteins from EPEC strains and one protein from a human strain designated as UPEC (upec-202, SAMN02802023), as well as eight animal strains. Additionally the genome encodes the intimin-like proteins FdeC and a SinH-variant. FHI_NMBU_03 was also positive for a cluster of the non-LEE-encoded effectors nleB, nleC, nleG, nleH and a frameshifted nleA pseudogene, located within a phage-region identified by PHASTER. Using CGE the MLST type was predicted to be ST28 and the fimH subtype was predicted to fimH90. A selection of chromosomal genes found by sequencing associated with virulence can be seen in Table 4. On the large virulence plasmid, ExPEC pathogenicity associated genes include: bor (an iss homologue), traT (serum resistance associated), the pyelonephritis-associated pilus pap operon; papABCDEFHJK, a putative pixG adhesin related gene encoding a protein $99 \%$ identical to a protein (EQZ28352.1) from the E. coli human UTI strain UMEA3585-1 (PRJNA186355), a putative autotransporter gene encoding an uncharacterized protein identical to protein EQZ28355.1 from UMEA- 3585-1, iroN (catecholate siderophore receptor), an AppA (HlyII) hemolysin protein and the leukotoxin genes $l k t B C D$.

The alkB gene coding for the alkylated DNA repair protein AlkB has an internal frameshift, and is probably inactive in FHI_NMBU_03. Several loci pertaining to fimbrial structures were found and noteworthy are genes related to K88-fimbria, 987P-fimbria and colonization factor antigen I fimbriae (CFA/I), which are all associated with ETEC strains. FHI_NMBU_03 is also positive for the YghJ protein gene, also known as SslE (Secreted and surface associated lipoprotein), which is a cell surface associated and secreted lipoprotein harbouring M60 metalloprotease domain [28]. 
Table 4 Selected virulence associated genes found on the FHI-NMBU-03 chromosome by nBLAST

\begin{tabular}{|c|c|c|c|c|c|}
\hline $\begin{array}{l}\text { Gene name } \\
\text { (FHI-NMBU-03 } \\
\text { chromosome) }\end{array}$ & Comment & Associated pathovar & $\%$ identity & Cover. & BLAST sequence \\
\hline$a a t B$ & $\begin{array}{l}\text { Autotransporter adhesin and virulence } \\
\text { factor of avian pathogenic Escherichia } \\
\text { coli. }\end{array}$ & APEC & 98.43 & $1017 / 1017$ & $J \times 402062$ \\
\hline herA & Archaeal bi-polar DNA helicase & Unknown & 99.47 & $1686 / 1686$ & NZ_NLRN01000019 \\
\hline$a s / A$ & Arylsulfatase gene & $\begin{array}{l}\text { ExPEC (Invasive K1 } \\
\text { strains) }\end{array}$ & 98.55 & $1656 / 1656$ & CU928163 \\
\hline aufC & Fimbrial usher protein gene & UPEC & 99.50 & $2595 / 2595$ & KE702411 \\
\hline $\operatorname{ces} A B$ & $\begin{array}{l}\text { Enteropathogenic Escherichia coli } \\
\text { chaperone for the type-III translocator } \\
\text { proteins }\end{array}$ & EPEC/STEC & 100.00 & $324 / 324$ & FM986651 \\
\hline $\operatorname{ces} D 2$ & $\begin{array}{l}\text { A second chaperone for the type III } \\
\text { secretion translocator protein EspD }\end{array}$ & EPEC/STEC & 98.28 & $407 / 408$ & NC013364 \\
\hline cest & $\begin{array}{l}\text { A bivalent enteropathogenic Escherichia } \\
\text { coli chaperone required for } \\
\text { translocation of both Tir and Map }\end{array}$ & EPEC/STEC & 100.00 & $471 / 471$ & LT903847 \\
\hline chuA & E.coli hemeutilization protein A gene & EXPEC & 99.65 & $1983 / 1983$ & LT827011 \\
\hline cif & Type III secreted effector & EPEC/STEC & 100.00 & 849 / 849 & AF497476 \\
\hline $\operatorname{csg} A$ & Major curlin subunit & Several & 99.56 & 459 / 459 & CP023388 \\
\hline $\operatorname{csg} B$ & Minor curlin subunit & Several & 99.56 & $456 / 456$ & СР027060 \\
\hline $\operatorname{csg} E$ & $\begin{array}{l}\text { Curli production assembly/transport } \\
\text { component }\end{array}$ & Several & 98.70 & $386 / 390$ & NC_011750 \\
\hline $\operatorname{csg} F$ & $\begin{array}{l}\text { Curli production assembly/transport } \\
\text { component }\end{array}$ & Several & 98.08 & $417 / 417$ & NC_011750 \\
\hline $\operatorname{csg} G$ & $\begin{array}{l}\text { Curli production assembly/transport } \\
\text { component }\end{array}$ & Several & 97.72 & 834 / 834 & CP003034 \\
\hline$c \vee a A$ & Colicin V secretion protein gene & Several & 100.00 & $1242 / 1242$ & GG773553 \\
\hline $\begin{array}{l}\text { Death on curing } \\
\text { RelE/ParE family toxin } \\
\text { gene }\end{array}$ & $\begin{array}{l}\text { Component of Toxin-antitoxin (TA) } \\
\text { system }\end{array}$ & Several & 100.00 & $272 / 276$ & CP023388 \\
\hline eae (subtype Beta2) & $\begin{array}{l}\text { Intimin - Necessary for the production of } \\
\text { attaching and effacing lesions on tissue } \\
\text { culture cells }\end{array}$ & EPEC/STEC & 100.00 & $2820 / 2820$ & AB647493 \\
\hline eсpA & Common pilus major fimbrillin subunit & Several & 98.47 & $588 / 588$ & BA000007 \\
\hline ecpD & $\begin{array}{l}\text { Fimbria adhesin of the E.coli common } \\
\text { pilus }\end{array}$ & Several & 99.64 & $1644 / 1644$ & СР019777 \\
\hline elfC & Putative fimbrial usher protein & Several & 99.73 & $2595 / 2595$ & CP021288 \\
\hline entA & Enterobactin biosynthesis gene & Several & 96.12 & $747 / 747$ & СР027060 \\
\hline entE & Enterobactin biosynthesis gene & Several & 95.65 & $1611 / 1611$ & СР027060 \\
\hline entH & Enterobactin biosynthesis gene & Several & 94.93 & $414 / 414$ & СР027060 \\
\hline escC & Outer membrane secretin & EPEC/STEC & 91.68 & $1539 / 1539$ & AP010958 \\
\hline escD & $\begin{array}{l}\text { Type III secretion system inner } \\
\text { membrane ring protein }\end{array}$ & EPEC/STEC & 99.10 & $1221 / 1221$ & BA000007 \\
\hline escF & $\begin{array}{l}\text { Type III secretion system needle major } \\
\text { subunit }\end{array}$ & EPEC/STEC & 100.00 & $222 / 222$ & NC_002695 \\
\hline escl & $\begin{array}{l}\text { Required for the formation of the type } \\
\text { III Secretion Apparatus }\end{array}$ & EPEC/STEC & 91.62 & $573 / 573$ & AP010958 \\
\hline $\operatorname{esc} N$ & Type III secretion ATPase & EPEC/STEC & 100.00 & $1341 / 1341$ & BA000007 \\
\hline$e s c R$ & $\begin{array}{l}\text { Type III secretion system export } \\
\text { apparatus protein gene }\end{array}$ & EPEC/STEC & 99.69 & $654 / 654$ & BA000007 \\
\hline escs & Type III secretion system export & EPEC/STEC & 100.00 & $270 / 270$ & BA000007 \\
\hline
\end{tabular}


Table 4 Selected virulence associated genes found on the FHI-NMBU-03 chromosome by nBLAST (Continued)

\begin{tabular}{|c|c|c|c|c|c|}
\hline $\begin{array}{l}\text { Gene name } \\
\text { (FHI-NMBU-03 } \\
\text { chromosome) }\end{array}$ & Comment & Associated pathovar & \% identity & Cover. & BLAST sequence \\
\hline & apparatus protein gene & & & & \\
\hline esct & $\begin{array}{l}\text { Type III secretion system export } \\
\text { apparatus protein gene }\end{array}$ & EPEC/STEC & 99.61 & $777 / 777$ & BA000007 \\
\hline escl & $\begin{array}{l}\text { Type III secretion system LEE export } \\
\text { apparatus switch protein gene }\end{array}$ & EPEC/STEC & 96.15 & $1038 / 1038$ & AP010958 \\
\hline escV & $\begin{array}{l}\text { Translocase of the type III secretion } \\
\text { system }\end{array}$ & EPEC/STEC & 99.70 & $2028 / 2028$ & BA000007 \\
\hline $\operatorname{esp} A$ & Type III secretions system gene & EPEC/STEC & 100.00 & $573 / 573$ & AJ225016 \\
\hline $\operatorname{espG}$ & $\begin{array}{l}\text { Type III secretion system effector, which } \\
\text { localize to the Golgi apparatus and } \\
\text { disrupt its architecture }\end{array}$ & EPEC/STEC & 98.41 & $1197 / 1197$ & BA000007 \\
\hline $\operatorname{etg} A$ & Lytic transglycosylase & EPEC/STEC & 100.00 & 459 / 459 & FM986650 \\
\hline fdec & $\begin{array}{l}\text { Mediates E. coli adhesion to mammalian } \\
\text { cells and extracellular matrix }\end{array}$ & EXPEC/STEC & 97.86 & $4251 / 4251$ & СР019777 \\
\hline $\mathrm{sfaH}$ & S-fimbrial protein subunit gene & EXPEC & 98.56 & $903 / 903$ & KT444704 \\
\hline$f l g D$ & $\begin{array}{l}\text { Flagellar basal body rod modification } \\
\text { protein gene }\end{array}$ & Several & 96.55 & $696 / 696$ & СР027060 \\
\hline$f l g M$ & Negative regulator of flagellin synthesis & Several & 98.97 & $290 / 294$ & CP028192 \\
\hline$f m / A$ & Major F9-fimbrial subunit & EXPEC/IPEC & 96.81 & $564 / 564$ & BA000007 \\
\hline fyuA & Ferric yersiniabactin uptake receptor & EXPEC & 99.51 & $2022 / 2022$ & СР016828 \\
\hline $\operatorname{gad}(1)$ & Glutamate decarboxylase gene & Several & 99.64 & $1401 / 1401$ & СР001671 \\
\hline $\operatorname{gad}(2)$ & Glutamate decarboxylase gene & Several & 99.79 & $1401 / 1401$ & FM180568 \\
\hline$g r / A$ & Global regulator of LEE activator & EPEC/STEC & 97.56 & $409 / 414$ & AP010958 \\
\hline gtrA & $\begin{array}{l}\text { Type IV O-antigen modification gene } \\
\text { (Shigella flexneri) }\end{array}$ & Unknown & 90.08 & $363 / 363$ & AF288197 \\
\hline hbp & $\begin{array}{l}\text { Hemoglobin-binding protease hbp } \\
\text { autotransporter gene }\end{array}$ & EXPEC & 99.95 & $4131 / 4131$ & CP009072 \\
\hline hlylll & $\begin{array}{l}\text { Gene encoding inner membrane } \\
\text { protein, hemolysin III family }\end{array}$ & EXPEC/IPEC & 98.41 & $690 / 690$ & СР003034 \\
\hline$i b e A$ & Invasion protein gene & NMEC/APEC/AIEC & 98.61 & $1371 / 1371$ & СР001855 \\
\hline$i b e B$ & Invasion protein gene & Several & 98.55 & $1383 / 1383$ & AF094824 \\
\hline ibeC/yijP/cptA & Invasion protein gene & Several & 99.77 & $1734 / 1734$ & СР019777 \\
\hline irpl & $\begin{array}{l}\text { HMWP1 nonribosomal peptide/ } \\
\text { polyketide synthase }\end{array}$ & EXPEC & 99.65 & $9492 / 9492$ & CU928163 \\
\hline irp2 & $\begin{array}{l}\text { HMWP2 Yersiniabactin biosynthetic } \\
\text { protein }\end{array}$ & EXPEC & 98.85 & $6106 / 6108$ & СР006834 \\
\hline ler & $\begin{array}{l}\text { Negative autoregulator of the LEE1 } \\
\text { operon }\end{array}$ & EPEC/STEC & 99.49 & $390 / 390$ & BA000007 \\
\hline $\operatorname{malx}$ & $\begin{array}{l}\text { Escherichia coli pathogenicity island- } \\
\text { marker }\end{array}$ & EXPEC & 98.61 & $1581 / 1581$ & AF003742 \\
\hline MAP & LEE effector protein gene & EPEC/STEC & 97.06 & $612 / 612$ & LC053401 \\
\hline $\begin{array}{l}\text { MBL-fold metallo } \\
\text { hydrolase gene }\end{array}$ & Putative phylogroup B2 specific marker & EXPEC & 99.52 & $1044 / 1044$ & СР023388 \\
\hline$m d t H$ & Multidrug resistance protein gene & Several & 99.83 & $1209 / 1209$ & СР019777 \\
\hline$m p c$ & Type III secretion system regulator gene & EPEC/STEC & 92.09 & $354 / 354$ & AP010953 \\
\hline mviM & Putative virulence factor & Several & 98.70 & 924 / 924 & CU928164 \\
\hline$n l e A^{a}$ & Non-LEE encoded effector A & EPEC/STEC & 99.84 & $1239 / 1239$ & AB303062 \\
\hline nleB & Non-LEE encoded effector B & EPEC/STEC & 100.00 & $981 / 981$ & AB303062 \\
\hline
\end{tabular}


Table 4 Selected virulence associated genes found on the FHI-NMBU-03 chromosome by nBLAST (Continued)

\begin{tabular}{|c|c|c|c|c|c|}
\hline $\begin{array}{l}\text { Gene name } \\
\text { (FHI-NMBU-03 } \\
\text { chromosome) }\end{array}$ & Comment & Associated pathovar & $\%$ identity & Cover. & BLAST sequence \\
\hline nlec-like gene & $\begin{array}{l}\text { T3SS secreted effector NleC-like protein } \\
\text { gene }\end{array}$ & EPEC/STEC & 100.00 & $264 / 264$ & CYEL01000033 \\
\hline nleG & Non-LEE encoded effector G & EPEC/STEC & 100.00 & $576 / 576$ & AB303062 \\
\hline nleH & Non-LEE encoded effector $\mathrm{H}$ & EPEC/STEC & 99.75 & $812 / 812$ & AP010958 \\
\hline usp/putative colicin & Uropathogenic specific protein gene & UPEC & 97.14 & $1782 / 1782$ & CU651637 \\
\hline $\operatorname{sep} L$ & Secretion switching protein gene & EPEC/STEC & 94.93 & $1046 / 1056$ & BA000007 \\
\hline $\operatorname{sep} Q$ & T3SS structure protein & EPEC/STEC & 95.53 & $918 / 918$ & СР003109 \\
\hline $\sin H$ & Intimin-like inverse autotransporter & EXPEC & 100.00 & $2178 / 2178$ & NZ_NMHI01000013 \\
\hline$s t c D$ & $\begin{array}{l}\text { Putative fimbrial-like adhesin protein } \\
\text { gene }\end{array}$ & IPEC & 99.71 & $1035 / 1035$ & NC_018658 \\
\hline$s t f D$ & Fimbrial protein gene & Unknown & 100.00 & $753 / 753$ & LOFW01000008 \\
\hline tir & $\begin{array}{l}\text { Translocated intimin receptor protein } \\
\text { gene }\end{array}$ & EPEC/STEC & 99.88 & $1650 / 1650$ & DQ206455 \\
\hline$x h / A$ & Xenorhabdus nematophila haemolysin & Unknown & 99.73 & $372 / 372$ & LDCR01000046 \\
\hline$y b t A$ & Yersiniabactin transcriptional regulator & EXPEC & 99.79 & $960 / 960$ & CP028714 \\
\hline yder & Fimbrial-like protein gene & Several & 98.41 & $504 / 504$ & CU928163 \\
\hline$y f c V$ & $\begin{array}{l}\text { Major subunit of a putative chaperone- } \\
\text { usher fimbria }\end{array}$ & EXPEC & 97.18 & $567 / 567$ & NC_011750 \\
\hline
\end{tabular}

${ }^{\text {a }}$ Frameshifted

A previously reported insertion of unknown origin with a base composition suggestive of horizontal gene transfer in a genetic region between mutS and rpoS, associated with phylogroup B2 and uropathogens [29] is additionally present. This region has later been named the $0454-n l p D$ region [30].

\section{Discussion}

Clinical microbiological laboratories and reference laboratories rely increasingly on genetic testing of faeces to identify possible pathogenic microbes. For enteric bacteria, a widely used practice is to perform PCR or real-time PCR assays, or other amplification methodology, to detect specific genes used for pathogen identification. For E. coli, PCR on faecal isolates [13] is used to detect the well-recognized IPEC pathovars EPEC, STEC, ETEC, EAEC and EIEC [31]. These pathovars all have genetic targets used for identification and classification. The most common genetic targets are the eae and $b f p$ genes for EPEC, stx 1 and stx 2 genes for STEC, genes encoding the thermostable (ST) and thermolabile (LT) toxins for ETEC, the $a g g R$ gene for EAEC, and the $i p a H$ gene for EIEC. These targets are also candidate targets for automatic pathogen identification systems, especially in a culture-independent diagnostic tests (CIDTs) workflow. The results from these assays will be a classification of the E. coli isolates into one of the recognized pathovars or, in case of no target amplification, a classification as a non-enteropathogenic or commensal strain.
In the present study, we looked at a wider range of virulence factors in faecal $E$. coli isolates submitted to the Reference Laboratory for Enteropathogenic Bacteria at the Norwegian Institute of Public Health (NIPH). We especially searched for known ExPEC VAGs as in recent years a heighten interest in the frequency of ExPEC strains in the human gut has emerged, however there are few studies examining the selection of VAGs used in the present study.

One surprising finding in our study was the high frequency of $E$. coli strains (64.3\%) with a combination of recognized IPEC and ExPEC VAGs. There are limited data on how common these IPEC/ExPEC hybrid strains are. In a study of $265 \mathrm{E}$. coli isolates from hospital inpatients and outpatients with UTIs, $10.6 \%$ of isolates harboured at least one IPEC virulence factor [32]. In previous studies of human faecal isolates, the E. coli strains are separately designated as IPEC or as commensal strains harbouring ExPEC VAGs, thus it is unclear how high of a percentage may be IPEC/ExPEC combinatory strains. The IPEC/ExPEC combination was especially high among the aEPEC strains (91.8\%).

One notable finding was that 13 out of 14 (92.9\%) ibeA positive isolates was an EPEC strains of phylogenetic group B2. Thus, ibeA carriage in faeces seems to be associated with a distinct group of IPEC strains in our material. The $i b e A$ gene is a known virulence factor of $E$. coli strains responsible for neonatal meningitis in humans (NMEC) by contributing to the invasion of 
brain microvascular endothelial cells (BMEC) [33]. It has also been described that ibeA plays an important role in the invasion of intestinal epithelial cells, as the absence of $i b e A$ accounted for a reduction in invasion of ca. 67\% compared to wild type in experiments with the adherent-invasive E. coli (AIEC) strain NRG857c and an ibeA deletion mutant strain (NRG857c $\Delta i b e A)$ [34]. Furthermore, ibeA was present in the genome of $26 \%$ of pathogenic isolates from chicken (APEC), but absent from the genome of non-pathogenic isolates of avian origin [35]. The ibeA gene was positively linked to the pathogenicity of the APEC strains, and it was additionally shown that $i b e A$ was involved in the invasion of human BMEC by the APEC strain BEN 2908 [35].

An interesting observation was the high number of strains harbouring genes coding for the trimeric autotransporter proteins (TAAs) EhaA and EhaG. Especially finding the ehaG gene in $48 \%$ of the strains with one or more ExPEC VAGs and no IPEC VAGs, since EhaG mediates specific adhesion to colorectal epithelial cells [36]. This indicates that $48 \%$ of our isolates carrying solely ExPEC VAGs may have the capacity to adhere to colorectal epithelial cells in humans. Both ehaA and ehaG are most prevalent in the phylogenetic groups B1 and D, while a difference between ehaA and ehaG was observed in phylogenetic group A where ehaA was not detected but ehaG was present in $34 \%$ of the isolates. The distribution pattern of ehaA and ehaG was in the same range as results from a study by Zude et al. 2014 [37], with the exception of phylogenetic group B2 where Zude et al. 2014 report that $21.9 \%$ of the strains carry the ehaG gene, while in the present study $7.1 \%$ of the B2 strains were positive for ehaG. EhaG is localized at the bacterial cell surface and, in addition to colorectal epithelial cell adhesion, promotes cell aggregation, biofilm formation, and adherence to a range of extracellular matrix (ECM) proteins [36]. TAAs are regarded as important virulence factors of many Gram-negative bacterial pathogens. We are aware that our PCR-based phylogrouping results may show minor differences from the 2013 Clermont method [38]. Non-IPEC strains are not stored at NIPH thus a re-typing of all strains using the 2013 Clermont method on all strains in this study is not possible, however the findings and conclusions are valid, and in future our phylogrouping will be sequenced-based e.g. by using online tools [39].

The fully sequenced FHI_NMBU_03 phylogroup B2 strain (with plasmid) from this study shows hallmarks of ExPEC pathovars UPEC, APEC, NMEC and the IPEC pathovar aEPEC with some VAGs related to ETEC (K88-, 987P- and CFA/I- fimbrial genes), thus it constitutes a truly pathovar-hybrid strain (Additional file 3). The eae gene alone will classify it as an aEPEC by most molecular diagnostics tests.
It was previously reported that YghJ caused extensive haemorrhage in mouse ileum in a dose dependent manner and it was suggested that YghJ could be a virulence factor of enteric pathogens associated with haemorrhagic diarrhoea [28]. A recent study additionally showed that the YghJ protein from a neonatal septicaemic E. coli altered cellular morphology of various cell lines and triggered the induction of several proinflammatory cytokines, which are attributed as one of the key mediators in the pathogenesis of sepsis [40].

Several factors classify this strain as UPEC (e.g. usp, $f y u A$, sfaS, the pap fimbrial operon, $\operatorname{chuA}$ and $y f(V)$. It has previously been reported that any two of $y f c V$, vat, or chuA along with $f y u A$ could be used to differentiate UPEC from diarrheagenic E. coli (DEC), human commensal, or animal commensal isolates. However, to differentiate UPEC from APEC, vat, fyuA, and $y f c V$ together are necessary, where the presence of the putative fimbrial subunit gene $y f c V$ is highly predictive of UPEC, increasing the odds of a strain being UPEC by 99.5 -fold [41].

The fimH90 subtype was also an interesting finding as it appears to be rare among $E$. coli strains and was not found among $243 \mathrm{draft}$ genomes of $E$. coli isolates in a study using the CGE FimTyper Web tool [42]. However, BLAST searches found an identical fim $H$ gene in a sequence scaffold from a human aEPEC strain (702898_aEPEC) isolated in Pakistan (GenBank: CYBW01000017.1). The CGE FimTyper confirmed this fimH gene to also be of subtype fimH90.

The comparison of sequence data with PCR typing revealed PCR positive results for $t s h$ and vat while sequencing showed the presence of the highly related $h b p$ gene on the chromosome and a putative related autotransporter on the virulence plasmid (locus tag: BXO92_24355). The PCR results can be explained by the similarity of the intended target genes, and the considerable confusion in GenBank submitted sequences on the correct nomenclature. The Tsh and Hbp proteins differ by only two amino acid residues. In addition, Vat and Tsh/Hbp are $77.5 \%$ identical in amino acids.

The plasmid located putative autotransporter protein (protein id: PRJNA362852:BXO92_24355) show $43.7 \%$ AA identity and $56.6 \%$ AA similarity to Tsh. RAST annotates this protein as EspC, while BASys annotates it as Hbp.

The number of GIs and integrated prophages indicate that FHI_NMBU_03 has obtained a high number of virulence factors by horizontal gene transfer and this may have been facilitated by a defect in the DNA-repair system with a frameshifted alkB gene. It is known that AlkB relevant lesions appear to represent strong blocks to replication, but these blocks can be bypassed by error-prone translesion DNA polymerases as a part of the SOS-system, leading to mutagenesis [43]. 
The $0454-n l p D$ region was shown to consist of several genetic patterns, where pattern III (the FHI_NMBU_03 sequence contains pattern III) had significant associations with phylogenetic group B2 strains, representing the most virulent members of the ExPEC group. This o454-nlpD region pattern was proposed as a tool to identify highly extraintestinal virulent strains among a mixed population of E. coli [30].

Strains closely related to FHI_NMBU_03 may have caused disease in Norway for an extended period of time as nine aEPEC intimin eae- $\beta 2$ carrying B2 strains of sequence type ST28 was previously detected among 56 aEPEC isolates from faecal specimens from children < 5 years old in Norway (five strains were from community-acquired diarrhoea samples) [44]. All nine strains where shown by microarray analysis to contain the ibeA, malX and usp genes as FHI_NMBU_03.

The high frequency of strains with combined IPEC/ ExPEC VAGs found in this study is worrisome as they might be capable of causing both intestinal- and extraintestinal disease. One scenario could be a general weakening of the immune system caused by ongoing intestinal disease, thereby creating an opportunity for spread of bacteria with ExPEC VAGs to other anatomical sites where the ExPEC VAGs may contribute to severe extraintestinal disease.

\section{Conclusion}

We report that a high frequency (>93\%) of routinely submitted faecal E. coli strains from Norwegian hospitals, previously characterized as IPEC, also harbour ExPEC virulence factors. Traditionally IPEC is regarded as a diarrhoeagenic pathogen with a set of virulence genes that is absent in ExPEC strains e.g. UPEC. This very high frequency of combined IPEC/ExPEC was an unexpected finding warranting further studies, as they may provide a rich source of opportunistic extraintestinal infections. WGS of one selected strain confirmed the pathovar-hybrid nature and revealed a genome heavily influenced by horizontal gene transfer (HGT). Sequence complex ST28 has previously been assigned to a hybrid group that was named "phylogroup ABD" [45], which supports our finding of the hybrid nature for strain FHI_NMBU_03.

\section{Additional files}

Additional file 1: $P C R$ primers used in study. Sequences of all $P C R$-primers used in this study, with references. (DOCX $18 \mathrm{~kb}$ )

Additional file 2: The Excel sheet contains VAGS PCR, Phylogenetic PCR and MLVA results for all $E$. coli strains included in this study. - PCR positive amplicons are listed as well as the MLVA profile and the results from the phylogenetic group PCR. (XLSX $21 \mathrm{~kb})$
Additional file 3: FHI-NMBU-03 SNPtree03 slanted. The image shows results from comparing the genome of $\mathrm{FHI}-\mathrm{NMBU}-03$ with a selection of E. coli whole genomes with E. coli K-12 MG1655 as reference. The SNP based phylogenetic tree was constructed using CSI Phylogeny 1.4 (https://cge.cbs.dtu.dk/services/CSIPhylogeny/). (PDF 10 kb)

\section{Abbreviations}

aEPEC: Atypical enteropathogenic Escherichia coli; AIEC: Adherent-invasive Escherichia coli; APEC: Avian pathogenic Escherichia coli; BMEC: Brain microvascular endothelial cells; CGE: Center for Genomic Epidemiology; cnf: Cytotoxic necrotising factor; DEC: Diarrheagenic Escherichia coli; E. coli: Escherichia coli; EAEC: Enteroaggregative Escherichia coli; EIEC: Enteroinvasive Escherichia coli; EnPEC: Endometrial pathogenic Escherichia coli; EPEC: Enteropathogenic Escherichia coli; ETEC: Enterotoxigenic Escherichia coli; ExPEC: Extraintestinal pathogenic Escherichia coli; Gls: Genomic islands; HGT: Horizontal gene transfer; IPEC: Intestinal pathogenic Escherichia coli; LEE: The locus of enterocyte effacement; MLST: Multilocus sequence typing; MLVA: Multiple Locus VariableNumber Tandem-Repeat Analysis; NIPH: Norwegian Institute of Public Health; NMEC: Neonatal meningitis Escherichia coli; NTEC: Necrotoxin producing Escherichia coli; ORFs: Open reading frames; SePEC: Septicaemic Escherichia coli; STEC: Shiga toxin producing Escherichia coli; TAAs: Trimeric autotransporter proteins; tEPEC: Typical enteropathogenic Escherichia coli; UPEC: Uropathogenic Escherichia coli; UTI: Urinary tract infection; VAGs: Virulence factors; WGS: Whole genome sequencing

\section{Acknowledgements}

We would like to thank Inger Løbersli and Marit Hindrum for technical help and support with strain characterization, MLVA, phylogeny and VAGS PCR-analysis.

\section{Funding}

No funding was obtained for this study.

\section{Availability of data and materials}

All results from PCR analyses (VAGs and Phylogeny) and MLVA genotyping is included in "Additional file 2" as an Excel spreadsheet. The datasets used and/or analysed during the current study are additionally available from the authors on reasonable request. All bacterial strains containing IPEC VAGs are available from the strain collection at the Norwegian Institute of Public Health, Oslo, Norway (https://www.fhi.no/en/more/access-to-data/), a fee might be applied. The nucleotide sequence of the hybrid strain FHI_NMBU_03 and its large virulence plasmid can be downloaded from NCBI's nucleotide database (https://www.ncbi.nlm.nih.gov/nucleotide/) with accession numbers NZ_CP019455.1 and NZ_CP019456.1 respectively.

\section{Authors' contributions}

BAL designed the project and participated in the experimental design, implementation and data analysis, wrote the first draft of the manuscript and was responsible for the final submission of the manuscript and submission of sequencing data to GenBank. MDF and DP contributed to WG-sequencing with MinION and MiSeq, data analysis pipeline (bioinformatics), annotations and strain comparisons. LTB participated in the experimental design, implementation and analysis of PCR results, strain selection and data analysis. All authors contributed to manuscript revisions. All authors have read and approved the final manuscript.

Ethics approval and consent to participate

Not applicable. The study did not involve human subjects, human material, nor human data.

\section{Consent for publication}

Not applicable. The study does not include details, images, or videos relating to any individual person.

\section{Competing interests}

The authors declare that they have no competing interests.

\section{Publisher's Note}

Springer Nature remains neutral with regard to jurisdictional claims in published maps and institutional affiliations. 


\section{Author details}

${ }^{1}$ Faculty of Chemistry, Biotechnology and Food Science, Norwegian University of Life Sciences, P.O. Box 5003, N-1432 Ås, Norway. ²Department of Zoonotic, Food- and Waterborne Infections, Norwegian Institute of Public Health, Oslo, Norway.

Received: 14 June 2018 Accepted: 16 October 2018

Published online: 01 November 2018

\section{References}

1. Chaudhuri RR, Henderson IR. The evolution of the Escherichia coli phylogeny. Infect Genet Evol. 2012;12(2):214-26.

2. Dale AP, Woodford N. Extra-intestinal pathogenic Escherichia coli (ExPEC): disease, carriage and clones. J Infect. 2015;71(6):615-26.

3. Johnson JR, Russo TA. Extraintestinal pathogenic Escherichia coli: "the other bad E coli". J Lab Clin Med. 2002;139(3):155-62.

4. Goldstone RJ, Popat R, Schuberth HJ, Sandra O, Sheldon IM, Smith DG. Genomic characterisation of an endometrial pathogenic Escherichia coli strain reveals the acquisition of genetic elements associated with extraintestinal pathogenicity. BMC Genomics. 2014;15:1075.

5. Sheldon IM, Rycroft AN, Dogan B, Craven M, Bromfield JJ, Chandler A, Roberts MH, Price SB, Gilbert RO, Simpson KW. Specific strains of Escherichia coli are pathogenic for the endometrium of cattle and cause pelvic inflammatory disease in cattle and mice. PLoS One. 2010:5(2):e9192.

6. Di Lorenzo M, Stork M. Plasmid-encoded Iron uptake systems. Microbiol Spectr. 2014:2(6). https://doi.org/10.1128/microbiolspec.PLAS-0030-2014.

7. Kaper JB, Nataro JP, Mobley HL. Pathogenic Escherichia coli. Nat Rev Microbiol. 2004;2(2):123-40.

8. Kohler CD, Dobrindt U. What defines extraintestinal pathogenic Escherichia Scoli? Int J Med Microbiol. 2011;301(8):642-7.

9. Luthje P, Brauner A. Virulence factors of uropathogenic E. coli and their interaction with the host. Adv Microb Physiol. 2014;65:337-72.

10. Starcic Erjavec M, Zgur-Bertok D. Virulence potential for extraintestinal infections among commensal Escherichia coli isolated from healthy humans--the Trojan horse within our gut. FEMS Microbiol Lett. 2015; 362(5). https://doi.org/10.1093/femsle/fnu061.

11. Karch H, Denamur E, Dobrindt U, Finlay BB, Hengge R, Johannes L, Ron EZ Tonjum T, Sansonetti PJ, Vicente M. The enemy within us: lessons from the 2011 European Escherichia coli O104:H4 outbreak. EMBO Mol Med. 2012; 4(9):841-8.

12. Jandhyala DM, Vanguri $V$, Boll EJ, Lai $Y$, McCormick BA, Leong JM. Shiga toxin-producing Escherichia coli O104:H4: an emerging pathogen with enhanced virulence. Infect Dis Clin N Am. 2013;27(3):631-49.

13. Brandal LT, Lindstedt BA, Aas L, Stavnes TL, Lassen J, Kapperud G. Octaplex PCR and fluorescence-based capillary electrophoresis for identification of human diarrheagenic Escherichia coli and Shigella spp. J Microbiol Methods. 2007;68(2):331-41.

14. Brandal LT, Sekse C, Lindstedt BA, Sunde M, Lobersli I, Urdahl AM, Kapperud G. Norwegian sheep are an important reservoir for human-pathogenic Escherichia coli O26:H11. Appl Environ Microbiol. 2012;78(12):4083-91.

15. Brandal LT, Wester AL, Lange H, Lobersli I, Lindstedt BA, Vold L, Kapperud G. Shiga toxin-producing escherichia coli infections in Norway, 1992-2012: characterization of isolates and identification of risk factors for haemolytic uremic syndrome. BMC Infect Dis. 2015;15:324.

16. Doumith M, Day MJ, Hope R, Wain J, Woodford N. Improved multiplex PCR strategy for rapid assignment of the four major Escherichia coli phylogenetic groups. J Clin Microbiol. 2012;50(9):3108-10.

17. Clermont $\mathrm{O}$, Bonacorsi $\mathrm{S}$, Bingen E. Rapid and simple determination of the Escherichia coli phylogenetic group. Appl Environ Microbiol. 2000;66(10):4555-8.

18. Lobersli I, Haugum K, Lindstedt BA. Rapid and high resolution genotyping of all Escherichia coli serotypes using 10 genomic repeat-containing loci. J Microbiol Methods. 2012;88(1):134-9.

19. Bolger AM, Lohse M, Usadel B. Trimmomatic: a flexible trimmer for Illumina sequence data. Bioinformatics. 2014;30(15):2114-20.

20. Watson M, Thomson M, Risse J, Talbot R, Santoyo-Lopez J, Gharbi K, Blaxter M. poRe: an $\mathrm{R}$ package for the visualization and analysis of nanopore sequencing data. Bioinformatics. 2015;31(1):114-5.

21. Bankevich A, Nurk S, Antipov D, Gurevich AA, Dvorkin M, Kulikov AS, Lesin VM, Nikolenko SI, Pham S, Prjibelski AD, et al. SPAdes: a new genome assembly algorithm and its applications to single-cell sequencing. J Comput Biol. 2012;19(5):455-77.
22. Angiuoli SV, Gussman A, Klimke W, Cochrane G, Field D, Garrity G, Kodira CD, Kyrpides N, Madupu R, Markowitz V, et al. Toward an online repository of standard operating procedures (SOPs) for (meta)genomic annotation. OMICS. 2008;12(2):137-41.

23. Van Domselaar GH, Stothard P, Shrivastava S, Cruz JA, Guo A, Dong X, Lu P, Szafron D, Greiner R, Wishart DS. BASys: a web server for automated bacterial genome annotation. Nucleic Acids Res. 2005;33(Web Server issue):W455-9.

24. Aziz RK, Bartels D, Best AA, DeJongh M, Disz T, Edwards RA, Formsma K, Gerdes S, Glass EM, Kubal M, et al. The RAST server: rapid annotations using subsystems technology. BMC Genomics. 2008;9:75.

25. Seemann T. Prokka: rapid prokaryotic genome annotation. Bioinformatics. 2014;30(14):2068-9.

26. Arndt D, Grant JR, Marcu A, Sajed T, Pon A, Liang Y, Wishart DS. PHASTER: a better, faster version of the PHAST phage search tool. Nucleic Acids Res. 2016;44(W1):W16-21.

27. Bertelli C, Laird MR, Williams KP, Simon Fraser University Research Computing G, Lau BY, Hoad G, Winsor GL, Brinkman FS. IslandViewer 4: expanded prediction of genomic islands for larger-scale datasets. Nucleic Acids Res. 2017:45(W1):W30-5.

28. Tapader R, Bose D, Pal A. YghJ, the secreted metalloprotease of pathogenic E. coli induces hemorrhagic fluid accumulation in mouse ileal loop. Microb Pathog. 2017;105:96-9.

29. Culham DE, Wood JM. An Escherichia coli reference collection group B2and uropathogen-associated polymorphism in the rpoS-mutS region of the E. coli chromosome. J Bacteriol. 2000;182(21):6272-6.

30. Ewers C, Dematheis F, Singamaneni HD, Nandanwar N, Fruth A, Diehl I, Semmler T, Wieler LH. Correlation between the genomic 0454-nlpD region polymorphisms, virulence gene equipment and phylogenetic group of extraintestinal Escherichia coli (ExPEC) enables pathotyping irrespective of host, disease and source of isolation. Gut Pathog. 2014;6:37.

31. Croxen MA, Law RJ, Scholz R, Keeney KM, Wlodarska M, Finlay BB. Recent advances in understanding enteric pathogenic Escherichia coli. Clin Microbiol Rev. 2013;26(4):822-80.

32. Toval F, Kohler CD, Vogel U, Wagenlehner F, Mellmann A, Fruth A, Schmidt MA, Karch H, Bielaszewska M, Dobrindt U. Characterization of Escherichia coli isolates from hospital inpatients or outpatients with urinary tract infection. J Clin Microbiol. 2014;52(2):407-18.

33. Huang $\mathrm{SH}$, Wan ZS, Chen YH, Jong AY, Kim KS. Further characterization of Escherichia coli brain microvascular endothelial cell invasion gene ibeA by deletion, complementation, and protein expression. J Infect Dis. 2001;183(7): 1071-8.

34. Cieza RJ, Hu J, Ross BN, Sbrana E, Torres AG. The IbeA invasin of adherentinvasive Escherichia coli mediates interaction with intestinal epithelia and macrophages. Infect Immun. 2015;83(5):1904-18.

35. Germon P, Chen YH, He L, Blanco JE, Bree A, Schouler C, Huang SH, MoulinSchouleur M. ibeA, a virulence factor of avian pathogenic Escherichia coli. Microbiology. 2005;151(Pt 4):1179-86.

36. Totsika M, Wells TJ, Beloin C, Valle J, Allsopp LP, King NP, Ghigo JM Schembri MA. Molecular characterization of the EhaG and UpaG trimeric autotransporter proteins from pathogenic Escherichia coli. Appl Environ Microbiol. 2012;78(7):2179-89.

37. Zude I, Leimbach A, Dobrindt U. Prevalence of autotransporters in Escherichia coli: what is the impact of phylogeny and pathotype? Int J Med Microbiol. 2014;304(3-4):243-56.

38. Clermont O, Christenson JK, Denamur E, Gordon DM. The Clermont Escherichia coli phylo-typing method revisited: improvement of specificity and detection of new phylo-groups. Environ Microbiol Rep. 2013;5(1):58-65.

39. Beghain J, Bridier-Nahmias A, Le Nagard H, Denamur E, Clermont O. ClermonTyping: an easy-to-use and accurate in silico method for Escherichia genus strain phylotyping. Microb Genom. 2018;4(7). https://doi.org/10.1099/ mgen.0.000192.

40. Tapader R, Bose D, Basu P, Mondal M, Mondal A, Chatterjee NS, Dutta P, Basu S, Bhadra RK, Pal A. Role in proinflammatory response of YghJ, a secreted metalloprotease from neonatal septicemic Escherichia coli. Int J Med Microbiol. 2016;306(7):554-65.

41. Spurbeck RR, Dinh PC Jr, Walk ST, Stapleton AE, Hooton TM, Nolan LK, Kim KS, Johnson JR, Mobley HL. Escherichia coli isolates that carry vat, fyuA, chuA, and yfc $V$ efficiently colonize the urinary tract. Infect Immun. 2012;80(12):4115-22.

42. Roer L, Tchesnokova V, Allesoe R, Muradova M, Chattopadhyay S, Ahrenfeldt J, Thomsen MCF, Lund O, Hansen F, Hammerum AM, et al. Development of a web tool for Escherichia coli subtyping based on fimH alleles. J Clin Microbiol. 2017;55(8):2538-43. 
43. Falnes PO, Klungland A, Alseth I. Repair of methyl lesions in DNA and RNA by oxidative demethylation. Neuroscience. 2007;145(4):1222-32.

44. Afset JE, Anderssen E, Bruant G, Harel J, Wieler L, Bergh K. Phylogenetic backgrounds and virulence profiles of atypical enteropathogenic Escherichia coli strains from a case-control study using multilocus sequence typing and DNA microarray analysis. J Clin Microbiol. 2008:46(7):2280-90.

45. Wirth T, Falush D, Lan RT, Colles F, Mensa P, Wieler LH, Karch H, Reeves PR, Maiden MCJ, Ochman H, et al. Sex and virulence in Escherichia coli: an evolutionary perspective. Mol Microbiol. 2006;60(5):1136-51.

Ready to submit your research? Choose BMC and benefit from:

- fast, convenient online submission

- thorough peer review by experienced researchers in your field

- rapid publication on acceptance

- support for research data, including large and complex data types

- gold Open Access which fosters wider collaboration and increased citations

- maximum visibility for your research: over $100 \mathrm{M}$ website views per year

At $\mathrm{BMC}$, research is always in progress.

Learn more biomedcentral.com/submissions 